\title{
Erratum: Star formation and dust attenuation properties in galaxies from a statistical ultraviolet-to-far-infrared analysis
}

\author{
by D. Burgarella, ${ }^{\star}$ V. Buat and J. Iglesias-Páramo
}

Key words: errata, addenda - galaxies: starburst - infrared: galaxies - ultraviolet: galaxies.

The paper 'Star formation and dust attenuation properties in galaxies from a statistical ultraviolet-to-far-infrared analysis' was published in Mon. Not. R. Astron. Soc. 360, 1413-1425 (2005).

Fig. 10 was incorrect in the paper. The labelling of the abscissa was wrong: it should be the best value for the amount of dust attenuation, i.e. $A_{\mathrm{FUV}}(+\mathrm{FIR})$ estimated using the far-infrared information. Moreover, the plotted points represented the opposite of the actual desired value, i.e. $A_{\mathrm{FUV}}(-\mathrm{FIR})-A_{\mathrm{FUV}}(+\mathrm{FIR})$ instead of the correct $A_{\text {FUV }}(+$ FIR $)-A_{\text {FUV }}(-\mathrm{FIR})$.

A corrected version of Fig. 10 is published here.

\section{REFERENCES}

Burgarella D., Buat V., Iglesias-Páramo J., 2005, MNRAS, 360, 1413

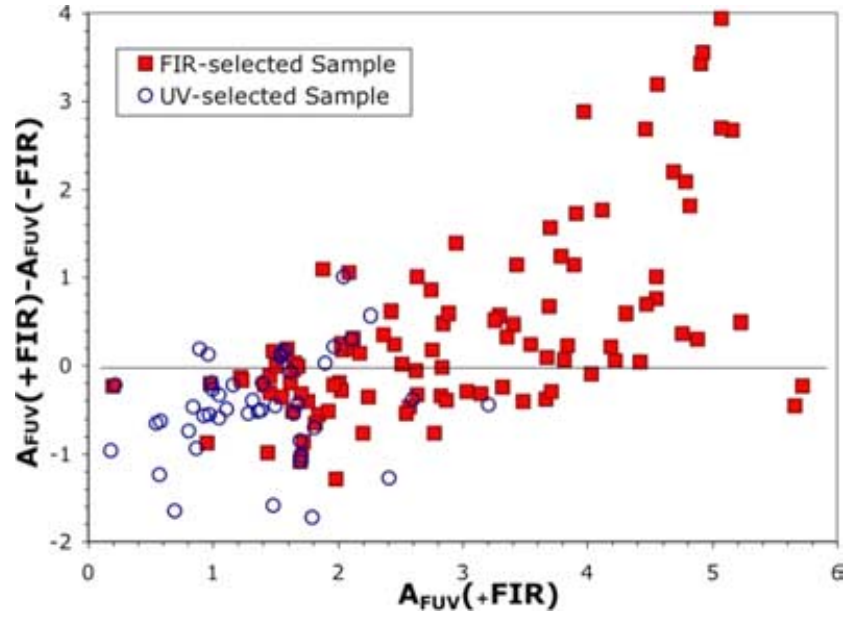

Figure 10. The FUV dust attenuation appears to be badly estimated for our UV-selected (blue) and FIR-selected (red) samples but the error is not uniformly distributed around 0 . The dust attenuation seems to be overestimated for the UV-selected sample and underestimated for the FIR-selected sample. This paper has been typeset from a $\mathrm{T}_{\mathrm{E}} \mathrm{X} / \mathrm{L} \mathrm{T}_{\mathrm{E}} \mathrm{X}$ file prepared by the author.

${ }^{\star}$ E-mail: denis.burgarella@oamp.fr 